\title{
Conservative Linear Difference Scheme for Rosenau-KdV Equation
}

\author{
Jinsong $\mathrm{Hu},{ }^{1}$ Youcai $\mathrm{Xu},{ }^{2}$ and Bing $\mathrm{Hu}^{2}$ \\ ${ }^{1}$ School of Mathematics and Computer Engineering, Xihua University, Chengdu 610039, China \\ ${ }^{2}$ School of Mathematics, Sichuan University, Chengdu 610064, China \\ Correspondence should be addressed to Youcai Xu; xyc@scu.edu.cn
}

Received 5 February 2013; Accepted 22 March 2013

Academic Editor: Hagen Neidhardt

Copyright (c) 2013 Jinsong Hu et al. This is an open access article distributed under the Creative Commons Attribution License, which permits unrestricted use, distribution, and reproduction in any medium, provided the original work is properly cited.

\begin{abstract}
A conservative three-level linear finite difference scheme for the numerical solution of the initial-boundary value problem of Rosenau-KdV equation is proposed. The difference scheme simulates two conservative quantities of the problem well. The existence and uniqueness of the difference solution are proved. It is shown that the finite difference scheme is of second-order convergence and unconditionally stable. Numerical experiments verify the theoretical results.
\end{abstract}

\section{Introduction}

$\mathrm{KdV}$ equation has been used in very wide applications and undergone research which can be used to describe wave propagation and spread interaction as follows [1-4]:

$$
u_{t}+u u_{x}+u_{x x x}=0 .
$$

In the study of the dynamics of dense discrete systems, the case of wave-wave and wave-wall interactions cannot be described using the well-known KdV equation. To overcome this shortcoming of the $\mathrm{KdV}$ equation, Rosenau $[5,6]$ proposed the so-called Rosenau equation:

$$
u_{t}+u_{x x x x t}+u_{x}+u u_{x}=0 .
$$

The existence and the uniqueness of the solution for (2) were proved by Park [7], but it is difficult to find the analytical solution for (2). Since then, much work has been done on the numerical method for (2) ([8-13] and also the references therein). On the other hand, for the further consideration of the nonlinear wave, the viscous term $+u_{x x x}$ needs to be included [14]

$$
u_{t}+u_{x x x x t}+u_{x}+u u_{x}+u_{x x x}=0 .
$$

This equation is usually called the Rosenau-KdV equation. Zuo [14] discussed the solitary wave solutions and periodic solutions for (2). Recently, [15-17] discussed the solitary solutions for the generalized Rosenau-KdV equation with usual power law nonlinearity. In $[15,16]$, the authors also gave the two invariants for the generalized Rosenau-KdV equation. In particular, [16] not only derived the singular 1-solition solution by the ansatz method but also used perturbation theory to obtain the adiabatic parameter dynamics of the water waves. In [17], The ansatz method is applied to obtain the topological soliton solution of the generalized Rosenau$\mathrm{KdV}$ equation. The $G^{\prime} / G$ method as well as the exp-function method are also applied to extract a few more solutions to this equation. But the numerical method to the initialboundary value problem of Rosenau-KdV equation has not been studied till now. In this paper, we propose a conservative three-level finite difference scheme for the Rosenau-KdV equation (3) with the boundary conditions

$$
\begin{gathered}
u\left(x_{L}, t\right)=u\left(x_{R}, t\right)=0, \quad u_{x}\left(x_{L}, t\right)=u_{x}\left(x_{R}, t\right)=0, \\
u_{x x}\left(x_{L}, t\right)=u_{x x}\left(x_{R}, t\right)=0, \quad t \in[0, T],
\end{gathered}
$$

and an initial condition

$$
u(x, 0)=u_{0}(x), \quad x \in\left[x_{L}, x_{R}\right]
$$


The initial boundary value problem (3)-(5) possesses the following conservative properties [15]:

$$
\begin{gathered}
Q(t)=\int_{x_{L}}^{x_{R}} u(x, t) d x=\int_{x_{L}}^{x_{R}} u_{0}(x) d x=Q(0), \\
E(t)=\|u\|_{L_{2}}^{2}+\left\|u_{x x}\right\|_{L_{2}}^{2}=E(0) .
\end{gathered}
$$

The solitary wave solution for $(3)$ is $[14,15]$

$$
\begin{aligned}
u(x, t)= & \left(-\frac{35}{24}+\frac{35}{312} \sqrt{313}\right) \\
\times \operatorname{sech}^{4}[ & \frac{1}{24} \sqrt{-26+2 \sqrt{313}} \\
& \left.\times\left(x-\left(\frac{1}{2}+\frac{1}{26} \sqrt{313}\right) t\right)\right] .
\end{aligned}
$$

When $-x_{L} \gg 0, x_{R} \gg 0$, the initial-boundary value problem (3)-(5) and the Cauchy problem (3) are consistent, so the boundary condition (4) is reasonable.

It is known the conservative scheme is better than the nonconservative ones. The nonconservative scheme may easily show nonlinear blow up. A lot of numerical experiments show that the conservative scheme can possesses some invariant properties of the original differential equation [18-29]. The conservative scheme is more suitable for longtime calculations. In [19], Li and Vu-Quoc said “... in some areas, the ability to preserve some invariant properties of the original differential equation is a criterion to judge the success of a numerical simulation." In this paper, we propose a three-level linear finite difference scheme for the Rosenau$\mathrm{KdV}$ equation (3)-(5). The difference scheme is conservative which simulates conservative properties (6) and (7) at the same time.

The rest of this paper is organized as follows. In Section 2, we propose a three-level linear finite difference scheme for the Rosenau-KdV equation and discuss the discrete conservative properties. In Section 3, we show that the scheme is uniquely solvable. Then, in Section 4, we prove that the finite difference scheme is of second-order convergence, unconditionally stable. Finally, some numerical tests are given in Section 5 to verify our theoretical analysis.

\section{Finite Difference Scheme and Conservation Properties}

Let $h=\left(x_{R}-x_{L}\right) / J$ and $\tau$ be the uniform step size in the spatial and temporal direction, respectively. Denote $x_{j}=x_{L}+j h(j=$ $-1,0,1,2, \ldots, J, J+1), t_{n}=n \tau(n=0,1,2, \ldots, N, N=$ $[T / \tau]), u_{j}^{n} \approx u\left(x_{j}, t_{n}\right)$ and $Z_{h}^{0}=\left\{u=\left(u_{j}\right) \mid u_{-1}=u_{0}=\right.$ $\left.u_{J}=u_{J+1}=0, j=-1,0,1,2, \ldots, J, J+1\right\}$. Throughout this paper, we denote $C$ as a generic positive constant independent of $h$ and $\tau$, which may have different values in different occurrences. We introduce the following notations:

$$
\begin{aligned}
& \left(u_{j}^{n}\right)_{x}=\frac{u_{j+1}^{n}-u_{j}^{n}}{h}, \quad\left(u_{j}^{n}\right)_{\bar{x}}=\frac{u_{j}^{n}-u_{j-1}^{n}}{h}, \\
& \left(u_{j}^{n}\right)_{\widehat{x}}=\frac{u_{j+1}^{n}-u_{j-1}^{n}}{2 h}, \quad\left(u_{j}^{n}\right)_{\hat{t}}=\frac{u_{j}^{n+1}-u_{j}^{n-1}}{2 \tau}, \\
& \bar{u}_{j}^{n}=\frac{u_{j}^{n+1}+u_{j}^{n-1}}{2}, \quad\left\langle u^{n}, v^{n}\right\rangle=h \sum_{j=1}^{J-1} u_{j}^{n} v_{j}^{n}, \\
& \left\|u^{n}\right\|^{2}=\left\langle u^{n}, u^{n}\right\rangle, \quad\left\|u^{n}\right\|_{\infty}=\max _{1 \leq j \leq J-1}\left\|u_{j}^{n}\right\| .
\end{aligned}
$$

We propose a three-level linear finite difference scheme for the solution of (3)-(5) as follows:

$$
\begin{gathered}
\left(u_{j}^{n}\right)_{\widehat{t}}+\left(u_{j}^{n}\right)_{x x \overline{x x} \hat{t}}+\left(\bar{u}_{j}^{n}\right)_{\widehat{x}}+\left(\bar{u}_{j}^{n}\right)_{x \bar{x} \hat{x}} \\
+\frac{1}{3}\left[u_{j}^{n}\left(\bar{u}_{j}^{n}\right)_{\widehat{x}}+\left(u_{j}^{n} \bar{u}_{j}^{n}\right)_{\widehat{x}}\right]=0, \\
j=1,2,3, \ldots, J-1, \quad n=1,2,3, \ldots, N-1, \\
u_{j}^{0}=u_{0}\left(x_{j}\right), \quad j=0,1,2,3, \ldots, J, \\
u^{n} \in Z_{h}^{0}, \quad\left(u_{0}^{n}\right)_{\widehat{x}}=\left(u_{J}^{n}\right)_{\widehat{x}}=0, \\
\left(u_{0}^{n}\right)_{x \bar{x}}=\left(u_{J}^{n}\right)_{x \bar{x}}=0, \quad n=1,2,3, \ldots, N .
\end{gathered}
$$

From the boundary conditions (4), we know that (13) is reasonable.

Lemma 1. It follows from summation by parts that for any two mesh functions $u, v \in Z_{h}^{0}$,

$$
\left\langle u_{x}, v\right\rangle=-\left\langle u, v_{x}\right\rangle, \quad\left\langle u_{x \bar{x}}, v\right\rangle=-\left\langle u_{x}, v_{x}\right\rangle .
$$

Then one has

$$
\left\langle u_{x}, u\right\rangle=-\left\langle u, u_{\bar{x}}\right\rangle, \quad\left\langle u_{x \bar{x}}, u\right\rangle=-\left\langle u_{x}, u_{x}\right\rangle=-\left\|u_{x}\right\|^{2} .
$$

Furthermore, if $\left(u_{0}\right)_{x \bar{x}}=\left(u_{J}\right)_{x \bar{x}}=0$, then

$$
\left\langle u_{x x \overline{x x}}, u\right\rangle=\left\|u_{x x}\right\|^{2} .
$$

The difference scheme (10)-(13) simulates two conservative properties of the problems (6) and (7) as follows.

Theorem 2. Suppose that $u_{0} \in H_{0}^{2}\left[x_{L}, x_{R}\right], u(x, t) \in C^{5,3}$, then the difference scheme (10)-(13) is conservative:

$$
\begin{gathered}
Q^{n}=\frac{h}{2} \sum_{j=1}^{J-1}\left(u_{j}^{n+1}+u_{j}^{n}\right)+\frac{h}{6} \tau \sum_{j=1}^{J-1} u_{j}^{n}\left(u_{j}^{n+1}\right)_{\widehat{x}}=Q^{n-1}=\cdots=Q^{0} \\
E^{n}=\frac{1}{2}\left(\left\|u^{n+1}\right\|^{2}+\left\|u^{n}\right\|^{2}\right)+\frac{1}{2}\left(\left\|u_{x x}^{n+1}\right\|^{2}+\left\|u_{x x}^{n}\right\|^{2}\right) \\
=E^{n-1}=\cdots=E^{0}
\end{gathered}
$$


Proof. Multiplying (10) with $h$, summing up for $j$ from 1 to $J-$ 1 , and considering the boundary condition (13) and Lemma 1, we get

$$
h \sum_{j=1}^{J-1} \frac{u_{j}^{n+1}-u_{j}^{n-1}}{2 \tau}+\frac{h}{6} \sum_{j=1}^{J-1}\left[u_{j}^{n}\left(u_{j}^{n+1}\right)_{\widehat{x}}-u_{j}^{n-1}\left(u_{j}^{n}\right)_{\widehat{x}}\right]=0 .
$$

Then, (17) is gotten from (19).

Taking an inner product of (10) with $2 \bar{u}^{n}$ (i.e., $u^{n+1}+u^{n-1}$ ), considering the boundary condition (13) and Lemma 1, we obtain

$$
\begin{aligned}
& \frac{1}{2 \tau}\left(\left\|u^{n+1}\right\|^{2}-\left\|u^{n-1}\right\|^{2}\right)+\frac{1}{2 \tau}\left(\left\|u_{x x}^{n+1}\right\|^{2}-\left\|u_{x x}^{n-1}\right\|^{2}\right) \\
& \quad+2\left\langle\bar{u}_{\widehat{x}}^{n}, \bar{u}^{n}\right\rangle+2\left\langle\bar{u}_{x \bar{x} \hat{x}}^{n}, \bar{u}^{n}\right\rangle \\
& \quad+2\left\langle P, \bar{u}^{n}\right\rangle=0
\end{aligned}
$$

where $P_{j}=(1 / 3)\left[u_{j}^{n}\left(\bar{u}_{j}^{n}\right)_{\widehat{x}}+\left(u_{j}^{n} \bar{u}_{j}^{n}\right)_{\widehat{x}}\right]$. According to

$$
\begin{gathered}
\left\langle\bar{u}_{\widehat{x}}^{n}, \bar{u}^{n}\right\rangle=0, \\
\left\langle\bar{u}_{x \bar{x} \widehat{x}}^{n}, \bar{u}^{n}\right\rangle=0, \\
\left\langle P, \bar{u}^{n}\right\rangle=\frac{1}{3} h \sum_{j=1}^{J-1}\left[u_{j}^{n}\left(\bar{u}_{j}^{n}\right)_{\widehat{x}}+\left(u_{j}^{n} \bar{u}_{j}^{n}\right)_{\widehat{x}}\right] \bar{u}_{j}^{n} \\
=\frac{1}{12} \sum_{j=1}^{J-1}\left[u_{j}^{n}\left(u_{j+1}^{n+1}+u_{j+1}^{n-1}-u_{j-1}^{n+1}-u_{j-1}^{n-1}\right)\right. \\
\left.+u_{j+1}^{n}\left(u_{j+1}^{n+1}+u_{j+1}^{n-1}\right)-u_{j-1}^{n}\left(u_{j-1}^{n+1}+u_{j-1}^{n-1}\right)\right] \\
\times\left(u_{j}^{n+1}+u_{j}^{n-1}\right) \\
=\frac{1}{12} \sum_{j=1}^{J-1}\left(u_{j}^{n}+u_{j+1}^{n}\right)\left(u_{j+1}^{n+1}+u_{j+1}^{n-1}\right)\left(u_{j}^{n+1}+u_{j}^{n-1}\right) \\
-\frac{1}{12} \sum_{j=1}^{J-1}\left(u_{j}^{n}+u_{j-1}^{n}\right)\left(u_{j}^{n+1}+u_{j}^{n-1}\right)\left(u_{j-1}^{n+1}+u_{j-1}^{n-1}\right) \\
=0,
\end{gathered}
$$

we have

$$
\left(\left\|u^{n+1}\right\|^{2}-\left\|u^{n-1}\right\|^{2}\right)+\left(\left\|u_{x x}^{n+1}\right\|^{2}-\left\|u_{x x}^{n-1}\right\|^{2}\right)=0 .
$$

Then, (18) is gotten from (23).

\section{Solvability}

Theorem 3. There exists $u^{n} \in Z_{h}^{0}$ which satisfies the difference scheme (10)-(13), $(1 \leq n \leq N)$.

Proof. Use mathematical induction to prove it. It is obvious that $u^{0}$ is uniquely determined by the initial condition (12).
We also can get $u^{1}$ in order $O\left(h^{2}+\tau^{2}\right)$ by two-level $C-N$ scheme (i.e., $u^{0}$ and $u^{1}$ are uniquely determined). Now suppose $u^{0}, u^{1}, \ldots, u^{n}(1 \leq n \leq N-1)$ is solved uniquely. Consider the equation of (10) for $u^{n+1}$ :

$$
\begin{aligned}
\frac{1}{2 \tau} u_{j}^{n+1} & +\frac{1}{2 \tau}\left(u_{j}^{n+1}\right)_{x x \bar{x} \bar{x}}+\frac{1}{2}\left(u_{j}^{n+1}\right)_{\widehat{x}} \\
& +\frac{1}{2}\left(u_{j}^{n+1}\right)_{x \bar{x} \hat{x}}+\frac{1}{6}\left[u_{j}^{n}\left(u_{j}^{n+1}\right)_{\widehat{x}}+\left(u_{j}^{n} u_{j}^{n+1}\right)_{\widehat{x}}\right]=0 .
\end{aligned}
$$

Taking an inner product of (24) with $u^{n+1}$, we get

$$
\begin{gathered}
\frac{1}{2 \tau}\left\|u^{n+1}\right\|^{2}+\frac{1}{2 \tau}\left\|u_{x x}^{n+1}\right\|^{2}+\frac{1}{2}\left\langle u_{\widehat{x}}^{n+1}, u^{n+1}\right\rangle+\frac{1}{2}\left\langle u_{x \bar{x} \widehat{x}}^{n+1}, u^{n+1}\right\rangle \\
+\frac{h}{6} \sum_{j=1}^{J-1}\left[u_{j}^{n}\left(u_{j}^{n+1}\right)_{\widehat{x}}+\left(u_{j}^{n} u_{j}^{n+1}\right)_{\widehat{x}}\right] u_{j}^{n+1}=0 .
\end{gathered}
$$

Similar to the proof of (21), we have

$$
\begin{aligned}
& \left\langle u_{\widehat{x}}^{n+1}, u^{n+1}\right\rangle=0, \\
& \left\langle u_{x \bar{x} \hat{x}}^{n+1}, u^{n+1}\right\rangle=0 .
\end{aligned}
$$

By

$$
\begin{aligned}
& \frac{h}{6} \sum_{j=1}^{J-1}\left[u_{j}^{n}\left(u_{j}^{n+1}\right)_{\widehat{x}}+\left(u_{j}^{n} u_{j}^{n+1}\right)_{\widehat{x}}\right] u_{j}^{n+1} \\
& =\frac{1}{12} \sum_{j=1}^{J-1}\left[u_{j}^{n}\left(u_{j+1}^{n+1}-u_{j-1}^{n+1}\right)+\left(u_{j+1}^{n} u_{j+1}^{n+1}-u_{j-1}^{n} u_{j-1}^{n+1}\right)\right] u_{j}^{n+1} \\
& =\frac{1}{12} \sum_{j=1}^{J-1}\left[u_{j}^{n} u_{j}^{n+1} u_{j+1}^{n+1}+u_{j+1}^{n} u_{j}^{n+1} u_{j+1}^{n+1}\right] \\
& \quad-\frac{1}{12} \sum_{j=1}^{J-1}\left[u_{j-1}^{n} u_{j-1}^{n+1} u_{j}^{n+1}+u_{j}^{n} u_{j-1}^{n+1} u_{j}^{n+1}\right]=0,
\end{aligned}
$$

and from (25)-(27), we have

$$
\left\|u^{n+1}\right\|^{2}+\left\|u_{x x}^{n+1}\right\|^{2}=0 .
$$

That is, (24) has only a trivial solution. Therefore, (10) determines $u_{j}^{n+1}$ uniquely. This completes the proof.

\section{Convergence and Stability}

Let $v(x, t)$ be the solution of problem (3)-(5), $v_{j}^{n}=v\left(x_{j}, t_{n}\right)$, then the truncation error of the difference scheme (10)-(13) is as follows:

$$
\begin{aligned}
r_{j}^{n}= & \left(v_{j}^{n}\right)_{\widehat{t}}+\left(v_{j}^{n}\right)_{x x \overline{x x} \widehat{t}}+\left(\bar{v}_{j}^{n}\right)_{\widehat{x}} \\
& +\left(\bar{v}_{j}^{n}\right)_{x \bar{x} \widehat{x}}+\frac{1}{3}\left[v_{j}^{n}\left(\bar{v}_{j}^{n}\right)_{\widehat{x}}+\left(v_{j}^{n} \bar{v}_{j}^{n}\right)_{\widehat{x}}\right] .
\end{aligned}
$$


Making use of Taylor expansion, we know that $r_{j}^{n}=O\left(\tau^{2}+h^{2}\right)$ holds if $h, \tau \rightarrow 0$.

Lemma 4. Suppose that $u_{0} \in H_{0}^{2}\left[x_{L}, x_{R}\right]$, then the solution $u^{n}$ of (3)-(5) satisfies

$$
\begin{array}{ll}
\|u\|_{L_{2}} \leq C, & \left\|u_{x}\right\|_{L_{2}} \leq C, \\
\|u\|_{L_{\infty}} \leq C, & \left\|u_{x}\right\|_{L_{\infty}} \leq C .
\end{array}
$$

Proof. It is follows from (7) that

$$
\|u\|_{L_{2}} \leq C, \quad\left\|u_{x x}\right\|_{L_{2}} \leq C
$$

By Holder inequality and Schwarz inequality, we get

$$
\begin{aligned}
\left\|u_{x}\right\|_{L_{2}}^{2} & =\int_{x_{L}}^{x_{R}} u_{x} u_{x} d x=\left.u u_{x}\right|_{x_{L}} ^{x_{R}}-\int_{x_{L}}^{x_{R}} u u_{x x} d x \\
& =-\int_{x_{L}}^{x_{R}} u u_{x x} d x \\
& \leq\|u\|_{L_{2}} \cdot\left\|u_{x x}\right\|_{L_{2}} \leq \frac{1}{2}\left(\|u\|_{L_{2}}^{2}+\left\|u_{x x}\right\|_{L_{2}}^{2}\right),
\end{aligned}
$$

which implies that

$$
\left\|u_{x}\right\|_{L_{2}} \leq C
$$

Using Sobolev inequality, we get that $\|u\|_{L_{\infty}} \leq C,\left\|u_{x}\right\|_{L_{\infty}} \leq$ C.

Lemma 5 (discrete Sobolev's inequality [27]). There exist two constants $C_{1}$ and $C_{2}$ such that

$$
\left\|u^{n}\right\|_{\infty} \leq C_{1}\left\|u^{n}\right\|+C_{2}\left\|u_{x}^{n}\right\| .
$$

Lemma 6 (discrete Gronwall inequality [27]). Suppose that $w(k)$ and $\rho(k)$ are nonnegative function and $\rho(k)$ is nondecreasing. If $C>0$, and

$$
w(k) \leq \rho(k)+C \tau \sum_{l=0}^{k-1} w(l), \quad \forall k,
$$

then

$$
w(k) \leq \rho(k) e^{C \tau k}, \quad \forall k
$$

Theorem 7. Suppose $u_{0} \in H_{0}^{2}\left[x_{L}, x_{R}\right]$, then the solution of (10)-(13) satisfies: $\left\|u^{n}\right\| \leq C,\left\|u_{x}^{n}\right\| \leq C,\left\|u_{x x}^{n}\right\| \leq C$, which yield $\left\|u^{n}\right\|_{\infty} \leq C,\left\|u_{x}^{n}\right\|_{\infty} \leq C(n=1,2, \ldots, N)$.

Proof. It is follows from (18) that

$$
\left\|u^{n}\right\| \leq C, \quad\left\|u_{x x}^{n}\right\| \leq C .
$$

According to (15) and Schwarz inequality, we get

$$
\left\|u_{x}^{n}\right\|^{2} \leq\left\|u^{n}\right\| \cdot\left\|u_{x x}^{n}\right\| \leq \frac{1}{2}\left(\left\|u^{n}\right\|^{2}+\left\|u_{x x}^{n}\right\|^{2}\right) \leq C .
$$

Using Lemma 5, we have $\left\|u^{n}\right\|_{\infty} \leq C,\left\|u_{x}^{n}\right\|_{\infty} \leq C$.
Theorem 8. Suppose $u_{0} \in H_{0}^{2}\left[x_{L}, x_{R}\right], u(x, t) \in C^{5,3}$, then the solution $u^{n}$ of the difference scheme (10)-(13) converges to the solution $v(x, t)$ of the problem (3)-(5) with order $\mathrm{O}\left(\tau^{2}+h^{2}\right)$ in norm $\|\cdot\|_{\infty}$.

Proof. Subtracting (10) from (29) and letting $e_{j}^{n}=v_{j}^{n}-u_{j}^{n}$, we have

$$
r_{j}^{n}=\left(e_{j}^{n}\right)_{\hat{t}}+\left(\bar{e}_{j}^{n}\right)_{x x \overline{x x \hat{t}}}+\left(\bar{e}_{j}^{n}\right)_{\widehat{x}}+\left(\bar{e}_{j}^{n}\right)_{x \bar{x} \widehat{x}}+R_{1, j}+R_{2, j},
$$

where $R_{1, j}=(1 / 3)\left[v_{j}^{n}\left(\bar{v}_{j}^{n}\right)_{\widehat{x}}-u_{j}^{n}\left(\bar{u}_{j}^{n}\right)_{\widehat{x}}\right], R_{2, j}=(1 / 3)\left[\left(v_{j}^{n} \bar{v}_{j}^{n}\right)_{\widehat{x}}-\right.$ $\left(u_{j}^{n} \bar{u}_{j}^{n}\right)_{\hat{x}}$. Computing the inner product of (39) with $2 \bar{e}^{n}$, we obtain

$$
\begin{aligned}
\left\langle r^{n}, 2 \bar{e}^{n}\right\rangle= & \frac{1}{2 \tau}\left(\left\|e^{n+1}\right\|^{2}-\left\|e^{n-1}\right\|^{2}\right)+\frac{1}{2 \tau}\left(\left\|e_{x x}^{n+1}\right\|^{2}-\left\|e_{x x}^{n-1}\right\|^{2}\right) \\
& +\left\langle\bar{e}_{\widehat{x}}^{n}, 2 \bar{e}^{n}\right\rangle+\left\langle\bar{e}_{x \bar{x} \hat{x}}^{n}, 2 \bar{e}^{n}\right\rangle+\left\langle R_{1}, 2 \bar{e}^{n}\right\rangle+\left\langle R_{2}, 2 \bar{e}^{n}\right\rangle .
\end{aligned}
$$

Similar to the proof of (21), we have

$$
\begin{gathered}
\left\langle\bar{e}_{\widehat{x}}^{n}, 2 \bar{e}^{n}\right\rangle=0, \\
\left\langle\bar{e}_{x \bar{x} \hat{x}}^{n}, 2 \bar{e}^{n}\right\rangle=0 .
\end{gathered}
$$

Then, (40) can be rewritten as follows:

$$
\begin{aligned}
& \left(\left\|e^{n+1}\right\|^{2}-\left\|e^{n+1}\right\|^{2}\right)+\left(\left\|e_{x x}^{n+1}\right\|^{2}-\left\|e_{x x}^{n-1}\right\|^{2}\right) \\
& \quad=2 \tau\left\langle r^{n}, 2 \bar{e}^{n}\right\rangle+2 \tau\left\langle-R_{1}, 2 \bar{e}^{n}\right\rangle+2 \tau\left\langle-R_{2}, 2 \bar{e}^{n}\right\rangle .
\end{aligned}
$$

Using Lemma 4 and Theorem 7, we get

$$
\begin{gathered}
\left|v_{j}^{n}\right| \leq C, \quad\left|u_{j}^{n}\right| \leq C, \\
\left|\left(u_{j}^{n}\right)_{\widehat{x}}\right| \leq C, \quad(j=0,1,2, \ldots, J ; n=1,2, \ldots, N) .
\end{gathered}
$$

According to the Schwarz inequality, we obtain

$$
\begin{aligned}
& \left\langle-R_{1}, 2 \bar{e}^{n}\right\rangle \\
& =-\frac{2}{3} h \sum_{j=1}^{J-1}\left[v_{j}^{n}\left(\bar{v}_{j}^{n}\right)_{\widehat{x}}-u_{j}^{n}\left(\bar{u}_{j}^{n}\right)_{\widehat{x}}\right] \bar{e}_{j}^{n} \\
& =-\frac{2}{3} h \sum_{j=1}^{J-1}\left[v_{j}^{n}\left(\bar{e}_{j}^{n}\right)_{\widehat{x}}+e_{j}^{n}\left(\bar{u}_{j}^{n}\right)_{\widehat{x}}\right] \bar{e}_{j}^{n} \\
& \leq \frac{2}{3} C h \sum_{j=1}^{J-1}\left(\left|\left(\bar{e}_{j}^{n}\right)_{\widehat{x}}\right|+\left|e_{j}^{n}\right|\right)\left|\bar{e}_{j}^{n}\right| \leq C\left[\left\|\bar{e}_{x}^{n}\right\|^{2}+\left\|e^{n}\right\|^{2}+\left\|\bar{e}^{n}\right\|^{2}\right] \\
& \leq C\left[\left\|e^{n+1}\right\|^{2}+\left\|e^{n-1}\right\|^{2}+\left\|e^{n}\right\|^{2}+\left\|e_{x}^{n+1}\right\|^{2}+\left\|e_{x}^{n-1}\right\|^{2}\right]
\end{aligned}
$$




$$
\begin{aligned}
& \left\langle-R_{2}, 2 \bar{e}^{n}\right\rangle \\
& =-\frac{2}{3} h \sum_{j=1}^{J-1}\left[\left(v_{j}^{n} \bar{v}_{j}^{n}\right)_{\widehat{x}}-\left(u_{j}^{n} \bar{u}_{j}^{n}\right)_{\widehat{x}}\right] \bar{e}_{j}^{n} \\
& =\frac{2}{3} h \sum_{j=1}^{J-1}\left[v_{j}^{n} \bar{v}_{j}^{n}-u_{j}^{n} \bar{u}_{j}^{n}\right]\left(\bar{e}_{j}^{n}\right)_{\widehat{x}} \\
& =\frac{2}{3} h \sum_{j=1}^{J-1}\left[v_{j}^{n} \bar{e}_{j}^{n}+e_{j}^{n} \bar{u}_{j}^{n}\right]\left(\bar{e}_{j}^{n}\right)_{\widehat{x}} \\
& \leq \frac{2}{3} C h \sum_{j=1}^{J-1}\left(\left|\left(\bar{e}_{j}^{n}\right)\right|+\left|e_{j}^{n}\right|\right)\left|\left(\bar{e}_{j}^{n}\right)_{\widehat{x}}\right| \\
& \leq C\left[\left\|\bar{e}_{x}^{n}\right\|^{2}+\left\|e^{n}\right\|^{2}+\left\|\bar{e}^{n}\right\|^{2}\right] \\
& \leq C\left[\left\|e^{n+1}\right\|^{2}+\left\|e^{n-1}\right\|^{2}+\left\|e^{n}\right\|^{2}+\left\|e_{x}^{n+1}\right\|^{2}+\left\|e_{x}^{n-1}\right\|^{2}\right] .
\end{aligned}
$$

Noting that

$$
\begin{aligned}
\left\langle r^{n}, 2 \bar{e}^{n}\right\rangle & =\left\langle r^{n}, e^{n+1}+e^{n-1}\right\rangle \\
& \leq\left\|r^{n}\right\|^{2}+\frac{1}{2}\left[\left\|e^{n+1}\right\|^{2}+\left\|e^{n-1}\right\|^{2}\right],
\end{aligned}
$$

and from (42)-(45), we have

$$
\begin{aligned}
\left(\left\|e^{n+1}\right\|^{2}\right. & \left.-\left\|e^{n-1}\right\|^{2}\right)+\left(\left\|e_{x x}^{n+1}\right\|^{2}-\left\|e_{x x}^{n-1}\right\|^{2}\right) \\
\leq C \tau & {\left[\left\|e^{n+1}\right\|^{2}+\left\|e^{n}\right\|^{2}+\left\|e^{n-1}\right\|^{2}\right.} \\
& \left.+\left\|e_{x}^{n+1}\right\|^{2}+\left\|e_{x}^{n}\right\|^{2}+\left\|e_{x}^{n-1}\right\|^{2}\right]+2 \tau\left\|r^{n}\right\|^{2} .
\end{aligned}
$$

Similar to the proof of (38), we have

$$
\begin{aligned}
\left\|e_{x}^{n+1}\right\|^{2} & \leq \frac{1}{2}\left(\left\|e^{n+1}\right\|^{2}+\left\|e_{x x}^{n+1}\right\|^{2}\right), \\
\left\|e_{x}^{n}\right\|^{2} & \leq \frac{1}{2}\left(\left\|e^{n}\right\|^{2}+\left\|e_{x x}^{n}\right\|^{2}\right), \\
\left\|e_{x}^{n-1}\right\|^{2} & \leq \frac{1}{2}\left(\left\|e^{n-1}\right\|^{2}+\left\|e_{x x}^{n-1}\right\|^{2}\right) .
\end{aligned}
$$

Then, (46) can be rewritten as

$$
\begin{aligned}
& \left(\left\|e^{n+1}\right\|^{2}-\left\|e^{n-1}\right\|^{2}\right)+\left(\left\|e_{x x}^{n+1}\right\|^{2}-\left\|e_{x x}^{n-1}\right\|^{2}\right) \\
& \leq C \tau\left[\left\|e^{n+1}\right\|^{2}+\left\|e^{n}\right\|^{2}+\left\|e^{n-1}\right\|^{2}\right. \\
& \left.+\left\|e_{x x}^{n+1}\right\|^{2}+\left\|e_{x x}^{n}\right\|^{2}+\left\|e_{x x}^{n-1}\right\|^{2}\right]+2 \tau\left\|r^{n}\right\|^{2} .
\end{aligned}
$$

Let $B^{n}=\left\|e^{n+1}\right\|^{2}+\left\|e_{x x}^{n+1}\right\|^{2}+\left\|e^{n}\right\|^{2}+\left\|e_{x x}^{n}\right\|^{2}$. Then, (48) can be rewritten as follows:

$$
B^{n}-B^{n-1} \leq C \tau\left(B^{n}+B^{n-1}\right)+2 \tau\left\|r^{n}\right\|^{2},
$$

which yields

$$
(1-C \tau)\left(B^{n}-B^{n-1}\right) \leq 2 C \tau B^{n-1}+2 \tau\left\|r^{n}\right\|^{2} .
$$

If $\tau$ is sufficiently small, which satisfies $1-C \tau>0$, then

$$
B^{n}-B^{n-1} \leq C \tau B^{n-1}+C \tau\left\|r^{n}\right\|^{2} .
$$

Summing up (51) from 1 to $n$, we have

$$
B^{n} \leq B^{0}+C \tau \sum_{l=1}^{n}\left\|r^{l}\right\|^{2}+C \tau \sum_{l=0}^{n-1} B^{l} .
$$

First, we can get $u^{1}$ in order $O\left(\tau^{2}+h^{2}\right)$ that satisfies $B^{0}=$ $O\left(\tau^{2}+h^{2}\right)^{2}$ by two-level $C-N$ scheme. Since

$$
\tau \sum_{l=1}^{n}\left\|r^{l}\right\|^{2} \leq n \tau \max _{1 \leq l \leq n}\left\|r^{l}\right\|^{2} \leq T \cdot O\left(\tau^{2}+h^{2}\right)^{2},
$$

then we obtain

$$
B^{n} \leq O\left(\tau^{2}+h^{2}\right)^{2}+C \tau \sum_{l=0}^{n-1} B^{l}
$$

From Lemma 6 we get

$$
B^{n} \leq O\left(\tau^{2}+h^{2}\right)^{2}
$$

which implies that

$$
\left\|e^{n}\right\| \leq O\left(\tau^{2}+h^{2}\right), \quad\left\|e_{x x}^{n}\right\| \leq O\left(\tau^{2}+h^{2}\right) .
$$

From (47) we have

$$
\left\|e_{x}^{n}\right\| \leq O\left(\tau^{2}+h^{2}\right) .
$$

By Lemma 5 we obtain

$$
\left\|e^{n}\right\|_{\infty} \leq O\left(\tau^{2}+h^{2}\right) .
$$

Finally, we can similarly prove results as follows.

Theorem 9. Under the conditions of Theorem 8, the solution $u^{n}$ of (10)-(13) is stable in norm $\|\cdot\|_{\infty}$.

\section{Numerical Simulations}

Since the three-level implicit finite difference scheme cannot start by itself, we need to select other two-level schemes (such as the $C-N$ Scheme) to get $u^{1}$. Then, be reusing initial value $u^{0}$, we can work out $u^{2}, u^{3}, \ldots$ Iterative numerical calculation is not required, for this scheme is linear, so it saves computing time. Let $x_{L}=-70, x_{R}=100$, and $T=40$,

$$
u_{0}(x)=\left(-\frac{35}{24}+\frac{35}{312} \sqrt{313}\right) \operatorname{sech}^{4}\left(\frac{1}{24} \sqrt{-26+2 \sqrt{313} x}\right) .
$$


TABLE 1: The error at various time steps.

\begin{tabular}{|c|c|c|c|c|c|c|}
\hline & \multicolumn{2}{|c|}{$\tau=h=0.1$} & \multicolumn{2}{|c|}{$\tau=h=0.05$} & \multicolumn{2}{|c|}{$\tau=h=0.025$} \\
\hline & $\left\|e^{n}\right\|$ & $\left\|e^{n}\right\|_{\infty}$ & $\left\|e^{n}\right\|$ & $\left\|e^{n}\right\|_{\infty}$ & $\left\|e^{n}\right\|$ & $\left\|e^{n}\right\|_{\infty}$ \\
\hline$t=10$ & $1.641934 e-3$ & $6.314193 e-4$ & $4.113510 e-4$ & $1.582641 e-4$ & $1.028173 e-4$ & $3.965867 e-5$ \\
\hline$t=20$ & $3.045414 e-3$ & $1.131442 e-3$ & $7.631169 e-4$ & $2.835874 e-4$ & $1.905450 e-4$ & $7.097948 e-5$ \\
\hline$t=30$ & $4.241827 e-3$ & $1.533771 e-3$ & $1.062971 e-3$ & $3.843906 e-4$ & $2.650990 e-4$ & $9.610332 e-5$ \\
\hline$t=40$ & $5.297873 e-3$ & $1.878952 e-3$ & $1.327645 e-3$ & $4.709118 e-4$ & $3.306738 e-4$ & $1.176011 e-4$ \\
\hline
\end{tabular}

TABLE 2: The verification of the second convergence.

\begin{tabular}{cccccc}
\hline & \multicolumn{3}{c}{$\left\|e^{n}(h, \tau)\right\| /\left\|e^{2 n}(h / 2, \tau / 2)\right\|$} & \multicolumn{2}{c}{$\left\|e^{n}(h, \tau)\right\|_{\infty} /\left\|e^{2 n}(h / 2, \tau / 2)\right\|_{\infty}$} \\
& $\tau=h=0.1$ & $\tau=h=0.05$ & $\tau=h=0.025$ & $\tau=h=0.1$ & $\tau=h=0.05$ \\
\hline$t=10$ & - & 3.991564 & 4.000797 & - & 3.989657 \\
$t=20$ & - & 3.990757 & 4.004916 & - & 3.989749 \\
$t=30$ & - & 3.990539 & 4.009713 & - & 3.990136 \\
$t=40$ & - & 3.990427 & 4.014970 & - & 3.990655 \\
\hline
\end{tabular}

TABLE 3: Numerical simulations on the two conservation invariants $Q^{n}$ and $E^{n}$.

\begin{tabular}{|c|c|c|c|c|c|c|}
\hline & \multicolumn{2}{|c|}{$\tau=h=0.1$} & \multicolumn{2}{|c|}{$\tau=h=0.05$} & \multicolumn{2}{|c|}{$\tau=h=0.025$} \\
\hline & $Q^{n}$ & $E^{n}$ & $Q^{n}$ & $E^{n}$ & $Q^{n}$ & $E^{n}$ \\
\hline$t=0$ & 5.497722548019 & 1.984553365290 & 5.498060684522 & 1.984390175264 & 5.498145418391 & 1.984349335263 \\
\hline$t=10$ & 5.497724936513 & 1.984595075859 & 5.498060837192 & 1.984401029470 & 5.498145479109 & 1.984352109750 \\
\hline$t=20$ & 5.497728744900 & 1.984645964099 & 5.498061080542 & 1.984414367496 & 5.498145545374 & 1.984355520610 \\
\hline$t=30$ & 5.497731963790 & 1.984679827211 & 5.498061287046 & 1.984423270337 & 5.498145609535 & 1.984357811266 \\
\hline$t=40$ & 5.497734235191 & 1.984701501262 & 5.498061398506 & 1.984428974030 & 5.498145659050 & 1.984359292230 \\
\hline
\end{tabular}

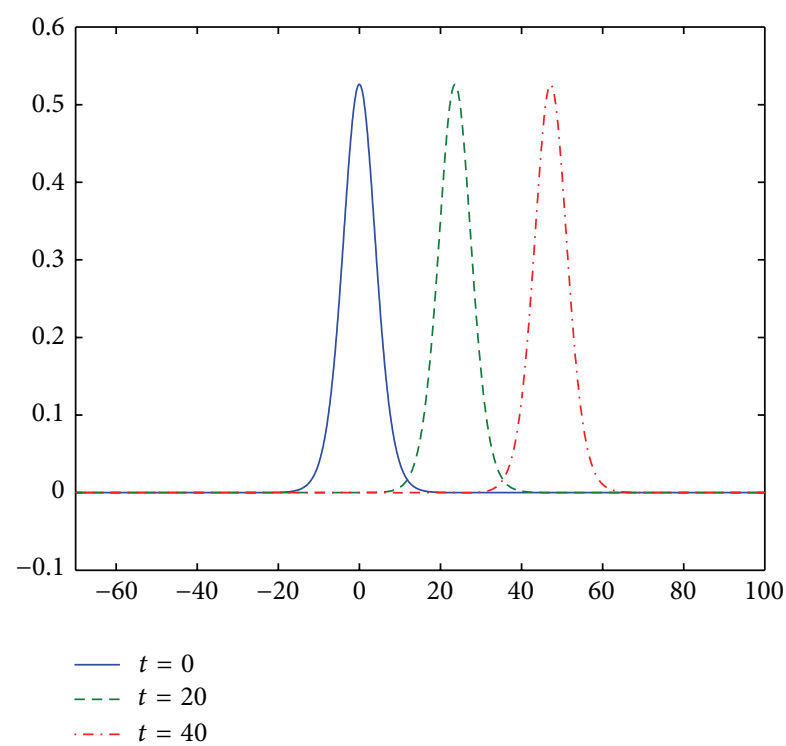

FIGURE 1: When $\tau=h=0.1$, the wave graph of $u(x, t)$ at various times.

In Table 1, we give the error at various time steps. Using the method in $[30,31]$, we verified the second convergence of the difference scheme in Table 2. Numerical simulations on two conservation invariants $Q^{n}$ and $E^{n}$ are given in Table 3 .

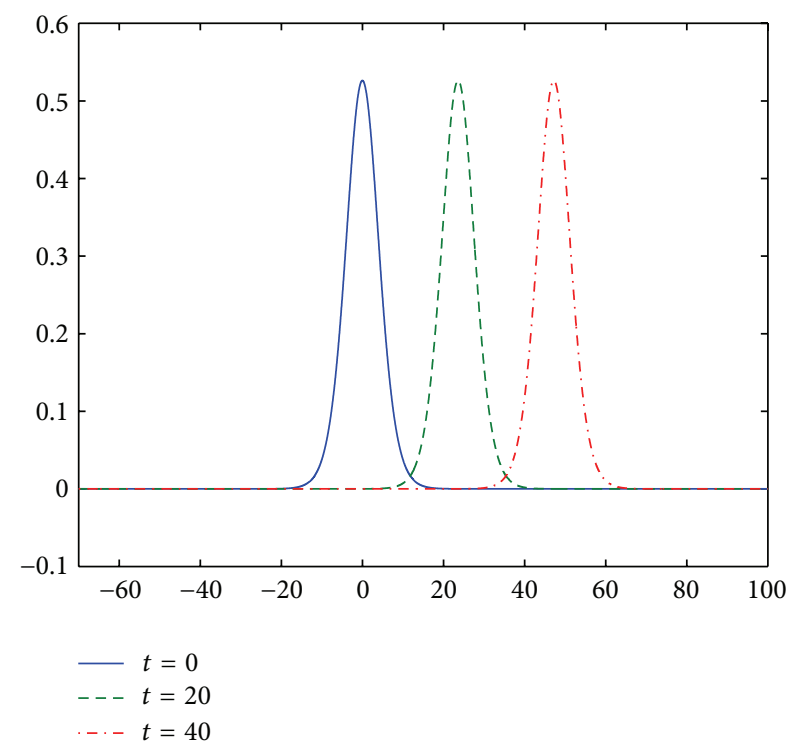

FigURE 2: When $\tau=h=0.025$, the wave graph of $u(x, t)$ at various times.

The wave graph comparison of $u(x, t)$ between $\tau=h=$ 0.1 and $\tau=h=0.025$ at various times is given in Figures 1 and 2 .

Numerical simulations show that the finite difference scheme is efficient. 


\section{Acknowledgments}

The work was supported by Scientific Research Fund of Sichuan Provincial Education Department (11ZB009) and the fund of Key Disciplinary of Computer Software and Theory, Sichuan, Grant no. SZD0802-09-1.

\section{References}

[1] Y. Cui and D.-k. Mao, "Numerical method satisfying the first two conservation laws for the Korteweg-de Vries equation," Journal of Computational Physics, vol. 227, no. 1, pp. 376-399, 2007.

[2] S. Zhu and J. Zhao, "The alternating segment explicit-implicit scheme for the dispersive equation," Applied Mathematics Letters, vol. 14, no. 6, pp. 657-662, 2001.

[3] A. R. Bahadır, "Exponential finite-difference method applied to Korteweg-de Vries equation for small times," Applied Mathematics and Computation, vol. 160, no. 3, pp. 675-682, 2005.

[4] S. Özer and S. Kutluay, "An analytical-numerical method for solving the Korteweg-de Vries equation," Applied Mathematics and Computation, vol. 164, no. 3, pp. 789-797, 2005.

[5] P. Rosenau, "A quasi-continuous description of a nonlinear transmission line," Physica Scripta, vol. 34, pp. 827-829, 1986.

[6] P. Rosenau, "Dynamics of dense discrete systems," Progress of Theoretical Physics, vol. 79, pp. 1028-1042, 1988.

[7] M. A. Park, "On the Rosenau equation," Matemática Aplicada e Computacional, vol. 9, no. 2, pp. 145-152, 1990.

[8] S. K. Chung and S. N. Ha, "Finite element Galerkin solutions for the Rosenau equation," Applicable Analysis, vol. 54, no. 1-2, pp. 39-56, 1994.

[9] K. Omrani, F. Abidi, T. Achouri, and N. Khiari, "A new conservative finite difference scheme for the Rosenau equation," Applied Mathematics and Computation, vol. 201, no. 1-2, pp. 3543, 2008.

[10] S. K. Chung, "Finite difference approximate solutions for the Rosenau equation," Applicable Analysis, vol. 69, no. 1-2, pp. 149156, 1998.

[11] S. K. Chung and A. K. Pani, "Numerical methods for the Rosenau equation," Applicable Analysis, vol. 77, no. 3-4, pp. 351369, 2001.

[12] S. A. V. Manickam, A. K. Pani, and S. K. Chung, "A second-order splitting combined with orthogonal cubic spline collocation method for the Rosenau equation," Numerical Methods for Partial Differential Equations, vol. 14, no. 6, pp. 695-716, 1998.

[13] Y. D. Kim and H. Y. Lee, "The convergence of finite element Galerkin solution for the Roseneau equation," The Korean Journal of Computational \& Applied Mathematics, vol. 5, no. 1, pp. 171-180, 1998.

[14] J.-M. Zuo, "Solitons and periodic solutions for the RosenauKdV and Rosenau-Kawahara equations," Applied Mathematics and Computation, vol. 215, no. 2, pp. 835-840, 2009.

[15] A. Esfahani, "Solitary wave solutions for generalized RosenauKdV equation," Communications in Theoretical Physics, vol. 55, no. 3, pp. 396-398, 2011.

[16] P. Razborova, H. Triki, and A. Biswas, "Perturbation of dispersive shallow water waves," Ocean Engineering, vol. 63, pp. 1-7, 2013.

[17] G. Ebadi, A. Mojaver, H. Triki, A. Yildirim, and A. Biswas, "Topological solitons and other solutions of the Rosenau-KdV equation with power law nonlinearity," Romanian Journal of Physics, vol. 58, no. 1-2, pp. 1-10, 2013.

[18] T. Wang, L. Zhang, and F. Chen, "Conservative schemes for the symmetric regularized long wave equations," Applied Mathematics and Computation, vol. 190, no. 2, pp. 1063-1080, 2007.

[19] S. Li and L. Vu-Quoc, "Finite difference calculus invariant structure of a class of algorithms for the nonlinear KleinGordon equation," SIAM Journal on Numerical Analysis, vol. 32, no. 6, pp. 1839-1875, 1995.

[20] Q. Chang, E. Jia, and W. Sun, "Difference schemes for solving the generalized nonlinear Schrödinger equation," Journal of Computational Physics, vol. 148, no. 2, pp. 397-415, 1999.

[21] T.-C. Wang and L.-M. Zhang, "Analysis of some new conservative schemes for nonlinear Schrödinger equation with wave operator," Applied Mathematics and Computation, vol. 182, no. 2, pp. 1780-1794, 2006.

[22] T. Wang, B. Guo, and L. Zhang, "New conservative difference schemes for a coupled nonlinear Schrödinger system," Applied Mathematics and Computation, vol. 217, no. 4, pp. 1604-1619, 2010.

[23] L. Zhang, "A finite difference scheme for generalized regularized long-wave equation," Applied Mathematics and Computation, vol. 168, no. 2, pp. 962-972, 2005.

[24] Z. Fei and L. Vázquez, "Two energy conserving numerical schemes for the sine-Gordon equation," Applied Mathematics and Computation, vol. 45, no. 1, pp. 17-30, 1991.

[25] Y. S. Wong, Q. Chang, and L. Gong, "An initial-boundary value problem of a nonlinear Klein-Gordon equation," Applied Mathematics and Computation, vol. 84, no. 1, pp. 77-93, 1997.

[26] Q. S. Chang, B. L. Guo, and H. Jiang, "Finite difference method for generalized Zakharov equations," Mathematics of Computation, vol. 64, no. 210, pp. 537-553, 1995.

[27] J. Hu and K. Zheng, "Two conservative difference schemes for the generalized Rosenau equation," Boundary Value Problems, Article ID 543503, 18 pages, 2010.

[28] X. Pan and L. Zhang, "On the convergence of a conservative numerical scheme for the usual Rosenau-RLW equation," Applied Mathematical Modelling, vol. 36, no. 8, pp. 3371-3378, 2012.

[29] X. Pan and L. Zhang, "Numerical simulation for general Rosenau-RLW equation: an average linearized conservative scheme," Mathematical Problems in Engineering, vol. 2012, Article ID 517818, 15 pages, 2012.

[30] T. Wang and B. Guo, "A robust semi-explicit difference scheme for the Kuramoto-Tsuzuki equation," Journal of Computational and Applied Mathematics, vol. 233, no. 4, pp. 878-888, 2009.

[31] B. Hu, J. Hu, and Y. Xu, "C-N difference schemes for dissipative symmetric regularized long wave equations with damping term," Mathematical Problems in Engineering, vol. 2011, Article ID 651642, 16 pages, 2011. 


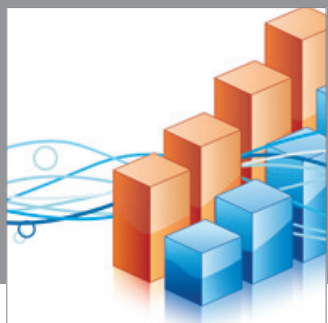

Advances in

Operations Research

mansans

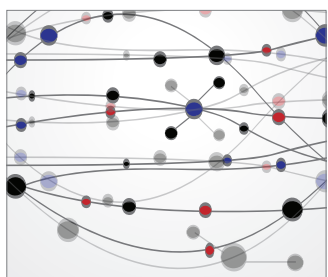

The Scientific World Journal
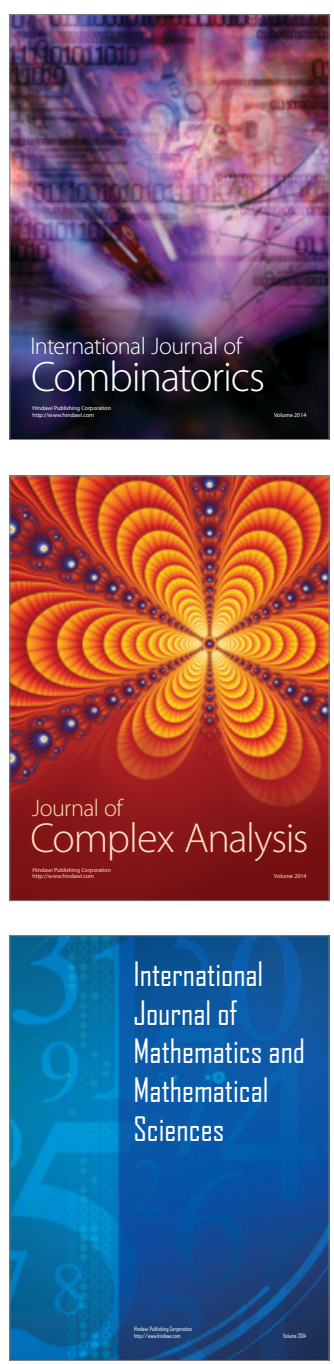
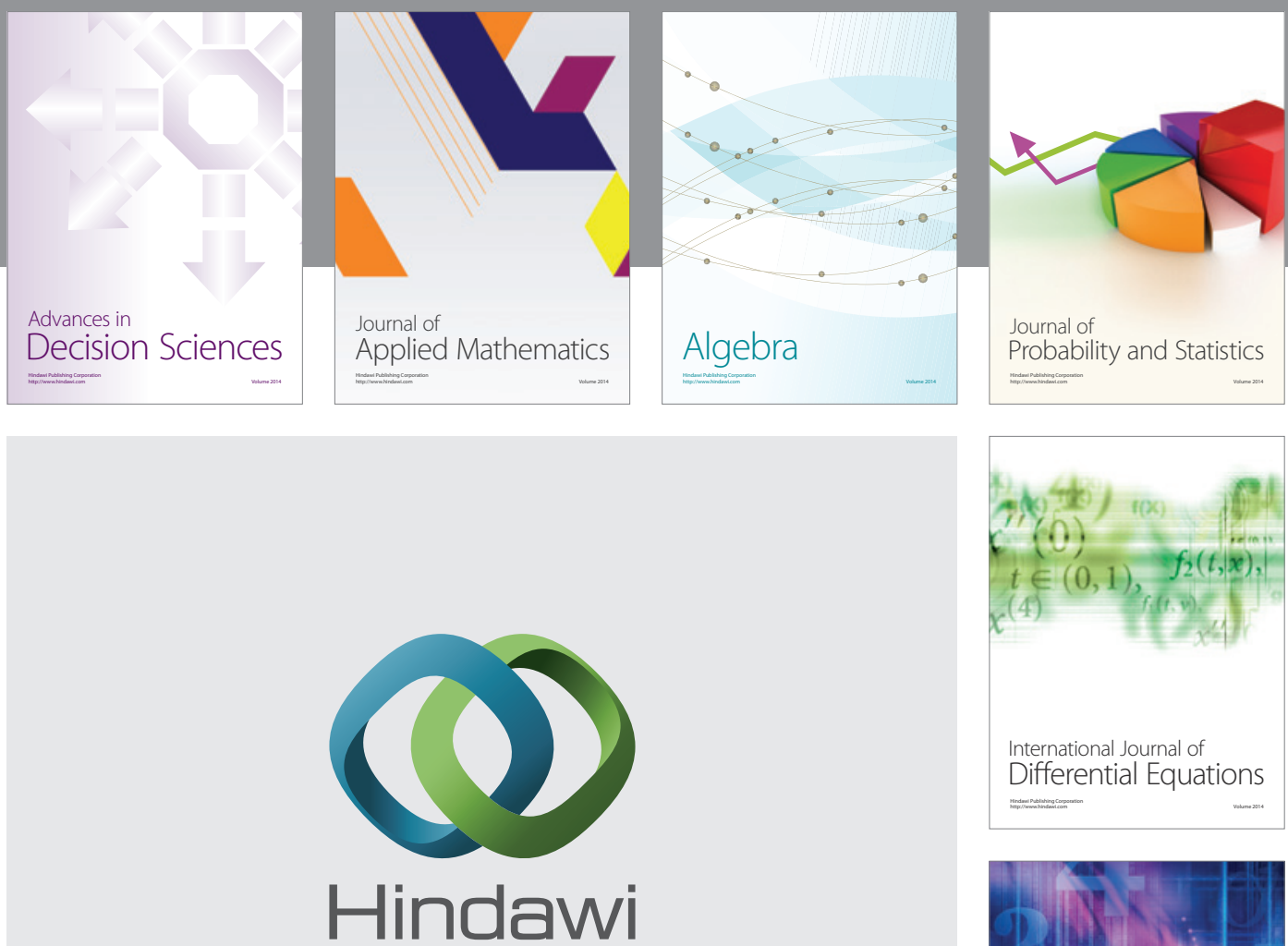

Submit your manuscripts at http://www.hindawi.com
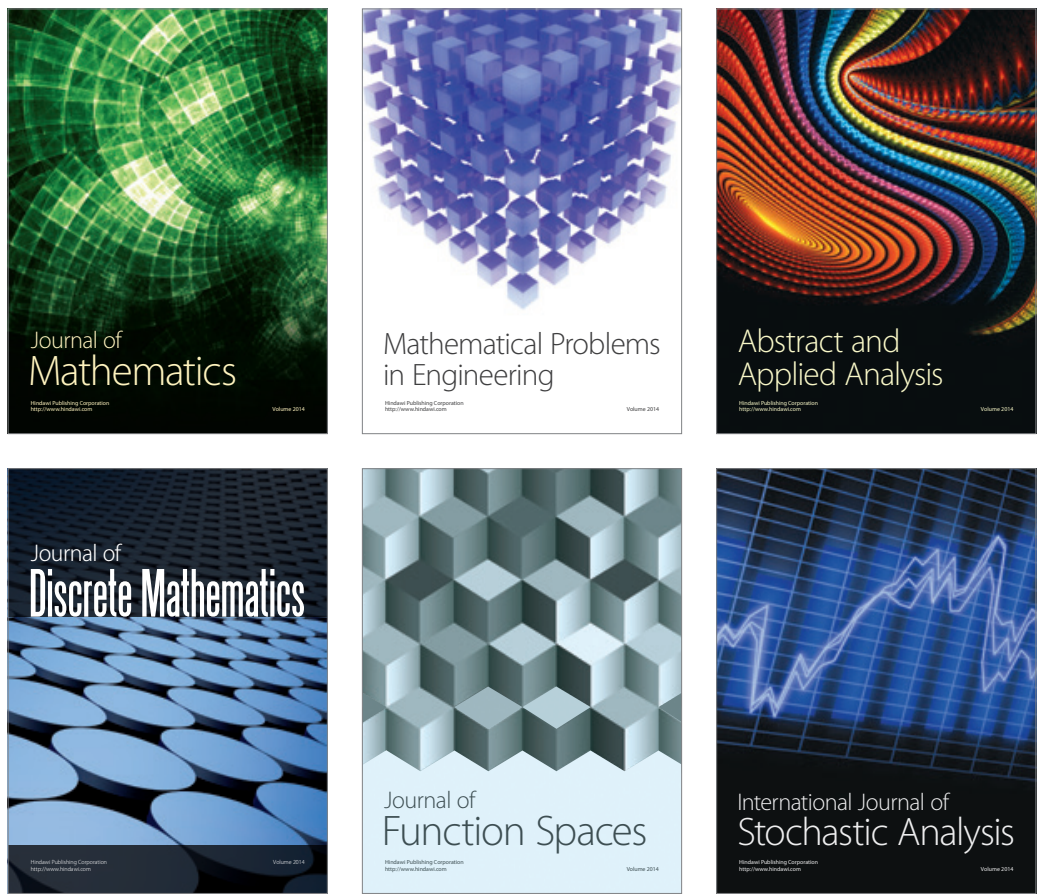

Journal of

Function Spaces

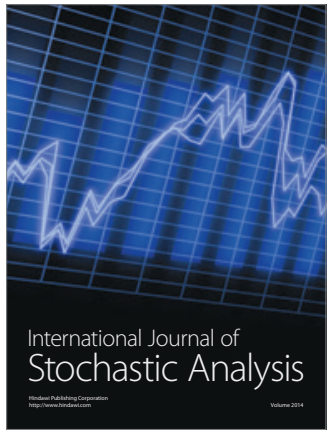

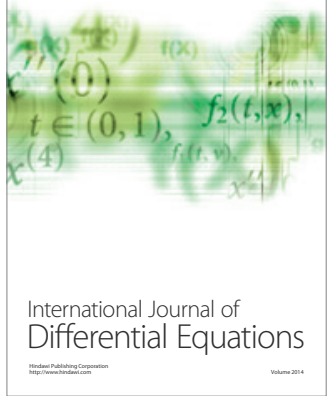
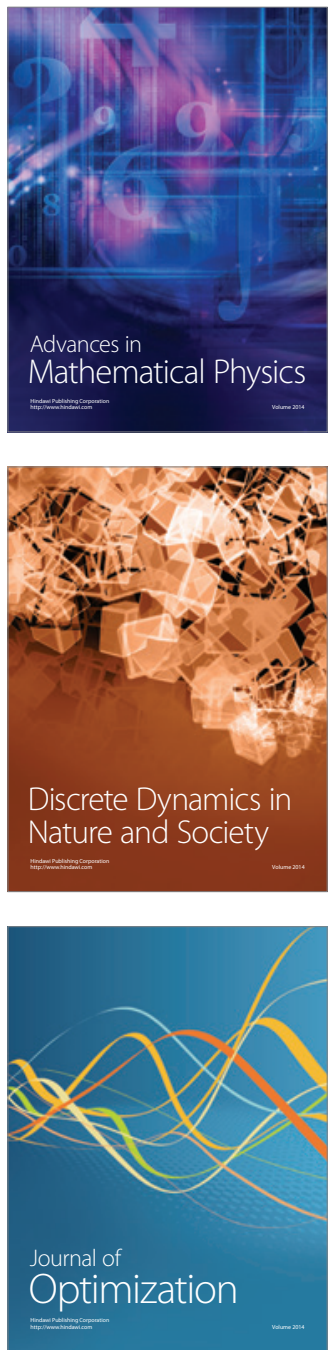